\title{
Four Years of Extreme Ultraviolet Observations of Markarian 421. I: Spectral Analysis
}

\author{
I. Cagnoni ${ }^{1,2}$ \\ and \\ A. Fruscione ${ }^{2}$ \\ ${ }^{1}$ SISSA, Via Beirut 4-34138, Trieste, Italy \\ ${ }^{2}$ Harvard-Smithsonian Center for Astrophysics, 60 Garden Street, Cambridge, MA 02138, USA
}

ilale@sissa.it

\begin{abstract}
We analyzed the $\sim 950 \mathrm{ks}$ of spectroscopic data accumulated by the Extreme Ultraviolet Explorer (EUVE) satellite between 1994 and 1997 for the BL Lacertae object Markarian 421. The EUV spectrum is well detected in the $70-110 \AA(112-177 \mathrm{eV})$ range and can be fitted by a power law model plus an absorption feature in the $\sim 71-75 \AA$ range. Previous studies of EUV absorption features in Mrk 421 and in the other EUV bright BL Lac object, PKS2155-304, explain this absorption feature as a superposition of Doppler-smeared absorption lines (mainly L- and M-shell transitions of $\mathrm{Mg}$ and $\mathrm{Ne}$ ) originating in high-velocity gas clouds ionized by the beamed continuum of the associated relativistic jet. We show that, for example, Fe IX L could also be a possibility consistent with the marginal detection of oxygen absorption lines in the Xray range. However physical models are highly sensitive to the assumptions on the photoionizing continuum and the surrounding gas.
\end{abstract}

Subject headings: galaxies: nuclei - galaxies: active - galaxies: individual (Mrk421)

\section{Introduction}

Evidence for absorption features in the X-ray spectra of BL Lac objects at $\sim 0.6 \mathrm{keV}^{1}$ has existed for many years. This is a tantalizing situation: BL Lac objects were originally defined by their lack of the sharp spectroscopic features that provide the normal tools for understanding astrophyisical objects. Without this tool, only large multisatellite, multitelescope campaigns spanning 20 decades of frequency have been able to constrain the BL Lac emission mechanism. Absorption features can provide a unique tool to study the presence and the dynamical properties of any gas surrounding the nucleus of the otherwise 'featureless' BL Lac objects. The extremely large Extreme Ultraviolet Explorer (EUVE) satellite

${ }^{1}$ PKS 2155-304 (Canizares \& Kruper 1984), H1426+428 (Sambruna et al. 1997) and PKS 0548-322 (Sambruna \& Mushotzky 1998) database on Mrk 421 offers a so far a unique opportunity to search for and characterize any sharp spectroscopic feature.

Mrk 421 is one of the brightest, best known and closest BL Lac objects $(z=0.0308$, Ulrich et al. 1975). Mrk 421 was in fact the first BL Lac object found to have X-ray emission (Richetts et al. 1976) and its low redshift makes this object one of the few extragalactic sources detected at $\gamma$ rays above $300 \mathrm{GeV}$ up to $\mathrm{TeV}$ energies (Punch et al. 1992). Mrk 421 has been extensively observed at radio (e.g. Zhang \& Baath 1990), UV/optical (Maza, Martin \& Angel 1978; Mufson et al., 1990) and X-ray frequencies (e.g. Mushotzky et al., 1979; George, Warwick \& Bromage 1988, Takahashi et al. 1996, Guainazzi et al. 1999). Mrk 421 shows optical polarization, a flat radio spectrum and significant time variability, characteristics of the blazar class (Makino et al., 1987) and its parent galaxy has been identified as a giant ellipti- 
cal (Ulrich et al. 1975; Mufson, Hutter \& Kondo 1989).

In the EUV band $(\sim 60-750 \AA)$ Mrk 421 was detected during the ROSATWFC survey (Pounds et al. 1993, Pye et al. 1995) and the EUVE allsky survey (Marshall, Fruscione \& Carone 1995, Fruscione 1996). It is one of the strongest EUV extragalactic sources and was immediately recognized as a prime target for subsequent EUV spectroscopic studies. For 4 almost consecutive years (1994, 1995, 1996 and 1998) Mrk 421 was the target of multiwavelength campaigns covering radio to TeV energies (Macomb et al., 1995, Kerrick et al. 1995, Buckley et al. 1996, Takahashi et al. 1999) and the EUVE satellite (which gives simultaneous photometric and spectroscopic data) was always part of the campaigns. In addition, in 1995 and in 1997 Mrk 421 was observed serendipitously with the EUVE photometers (the "scanners").

The large amount of data accumulated for Mrk 421 by the $E U V E$ satellite to date $(\sim 1100 \mathrm{ks}$ of public imaging data and $\sim 950 \mathrm{ks}$ of public spectroscopic data) represents the best coverage for any BL Lac object at high energies. Less than half of this data has been analysed to date (Fruscione et al. 1996, Kartje et al. 1997, hereafter K97) revealing that (i) the source exhibits a flare-like behavior (ii) the strong EUV variability is correlated with soft Xray and $\mathrm{TeV}$-energy emission, and (iii) the spectrum may have strong absorption features between $65 \AA$ and $75 \AA$ (0.16-0.19 keV) (K97). However in the EUV, all the previous analysis of BL Lacs can only infer the presence of absorption from the extrapolation of the X-ray spectrum (e.g. Konigl et al 1995 for PKS2155-304 and K97 for MRK 421).

The purpose of this paper is to analyze, in an homogeneous way, all of the $950 \mathrm{ks}$ of EUVE public spectroscopic data for Mrk 421 . The results of this paper will also provide a useful reference for the calibration of other satellites observing in this energy range. In particular Mrk 421 is a calibration target for both the Chandra and XMM satellites, whose Low Energy Transmission Grating (LETG) and Reflection Grating Spectrometer (RGS) partially overlap with the EUVE energy band.

In Section 2 we present all the EUVE spectra of Mrk 421 taken from 1994 to 1997; in Section 3 we discuss the results and in Section 4 we give a brief summary and our conclusions. All the photomet- ric data, the corresponding lightcurves and a detailed variability analysis are presented in a companion paper (Cagnoni, Papadakis \& Fruscione, 2000, hereafter paper II).

\section{Observations}

The EUVE satellite was launched in June 1992 and is still acquiring astronomical data in the wavelength range from $\sim 60$ to $750 \AA$. Onboard EUVE there are 4 telescopes: three of them are co-aligned photometers observing in 4 different EUV bandpasses, while the fourth is the Deep Survey/Spectrometer (DS/S, see e.g. Welsh et al. 1990) mounted orthogonally to the scanners. (Figure 1 of paper II shows the DS effective area compared to the scanner effective area). In addition to a photometer (10\% bandpass $68-178 \AA$ ) the DS/S telescope is also equipped with three spectrometers (Hettrick \& Bowyer, 1983; Abbott et al. 1996) covering the "short" (SW: $70-190 \AA$ ), "medium" (MW: $140-380 \AA$ ) and "long" (LW: $280-760 \AA$ ) EUV wavelengths. This configuration allows simultaneous imaging and spectroscopy with a spatial resolution of $\sim 1^{\prime}$ and a spectral resolution of $\lambda / \Delta \lambda \sim 200$ at the short wavelengths, i.e. $0.35 \AA$ at $70 \AA$.

Further details on the science instrumentation and performances can be found in Malina (1994), Sirk et al. (1997) and Abbott et al. (1996).

The scanners were used to carry out the EUVE all-sky survey (July 211992 - January 23 1993) and are currently utilized in the Right Angle Program (RAP), that is the observation of targets of scientific interest with the scanner telescopes while the DS/S telescope is conducting the primary science observation. The DS/S has been used for a deep EUV survey along the ecliptic during the all-sky survey and for pointed spectroscopic and imaging observations since then.

We use throughout this paper all of the publicly available DS/S data on MRK 421: from 1994 to 1997 , a total of $950 \mathrm{ks}$. We are not considering the most recent observation (April 1998) since the data is presently being analysed by the original proposers (Marshall et al. in preparation). Mrk 421 was observed by EUVE several times from 1994 to 1997, four times with the DS/S and twice with the scanners; Table 1 summarizes the observations. 
Because of strong absorption by the interstellar medium (ISM) along the line of sight (even at the low $N_{H}=1.45 \times 10^{20} \mathrm{~cm}^{-2}$ Elvis, Wilkes \& Lockman 1989), Mrk 421 was detected only at the shortest wavelengths: in the Lex/B filter of the scanners ( $10 \%$ bandpass $58-174 \AA$ ), in the Lex/B filter of the DS instrument (10\% bandpass 67-178 $\AA$ ) and, relevant for this paper, in the SW spectrometer $(70-190 \AA)^{2}$.

\section{1. $\quad$ Energy spectra}

After rejecting the time intervals with high particle background, such as the satellite passages over the South Atlantic Anomaly, and correcting the data for instrumental deadtime and telemetry saturation, we measured a total effective exposure of $315858 \mathrm{~s}, 506780 \mathrm{~s}$, and $354806 \mathrm{~s}$ for the 1994 , 1995 and 1996 observations respectively (note that the first 1995 and the 1997 observations were performed with the scanners, instrument with imaging capabilities only).

We extracted all the spectra in a homogeneous way from the two-dimensional SW detector image, using the EUVE Guest Observer Center software (IRAF/EUV package) and other standard spectroscopic IRAF tasks. The spectrum was extracted in a 14 pixel wide aperture and the background obtained by linearly fitting an average background in two regions ( $\sim 80$ pixel wide) of the detector, one on each side of the spectrum. The resulting wavelength-calibrated spectra (Figures 1, 4 and 8) were convolved with the proper effective area and binned over $1 \AA$. For the 1994 on-axis observation (Figure 1) we used the on-axis effective area derived from in-orbit calibrations for the $75 \AA$ - $110 \AA$ range. The 1995 and 1996 observations were performed off-axis (a technique that slightly increases the short-wavelength coverage, down to $\sim 70 \AA$ ). This results in a different effective area (Marshall et al. 1999) that we applied here for the first time. The $1 \sigma$ error bars on the flux measurements shown in the figures of all the spectra were computed us-

\footnotetext{
${ }^{2} \mathrm{~A}$ graphical representation of the simulated DS/S and scanner responses is presented in Figure 2 of paper II, where we convolved the Mrk 421 energy spectrum corrected for the ISM absorption with the DS/S and scanners effective area. No signal is present at wavelengths longer than $\sim 110 \AA$
}

ing the formula

$$
\sigma(\lambda)=\frac{\sqrt{\left[S_{\lambda}+B_{\lambda} /\left(1+H_{s p} / H_{B}\right)\right]}}{A_{e f f}(\lambda)}
$$

where $\mathrm{S}$ is the signal from the target, $\mathrm{B}$ the averaged background, both in counts/s, $A_{\text {eff }}$ the SW effective area in $\mathrm{cm}^{2}$ and $H_{s p}$ and $H_{B}$ are respectively the width in pixels of the extraction region for spectrum (14 pixels) and background (160 pixels) in the direction perpendicular to the dispersion.

We modeled the EUV spectrum of Mrk 421 using version 2.0 of the SHERPA modelling and fitting software (recently developed at the Chandra X-ray Center). We initially used an absorbed power law of the form

$\mathrm{f}(\lambda)=\mathrm{f}\left(\lambda_{0}\right) \lambda^{\alpha-1} \exp \left\{-\left[\Sigma_{\mathrm{X}} \mathrm{N}(\mathrm{X}) \sigma_{\mathrm{X}}\right)\right\} \quad$ photons $\mathrm{cm}^{-2} \mathrm{~s}^{-2} \AA^{-1}$

where $\mathrm{f}\left(\lambda_{0}\right)$ is a normalization factor. We used $\lambda_{0}=80 \AA\left(\mathrm{E}_{0}=0.155 \mathrm{keV}\right) . \alpha$ is the energy index and the absorption is characterized by a column density $\mathrm{N}(\mathrm{X})$ and an absorption cross section $\sigma_{\mathrm{X}}$ for each element. We included in the model $\mathrm{H}, \mathrm{He}$ I and He II (Rumph, Bowyer and Vennes, 1994) and heavier elements (Morrison and McCammon, 1983). We fixed the Galactic hydrogen column density at $N_{H}=1.45 \times 10^{20} \mathrm{~cm}^{-2}$ (Elvis, Wilkes \& Lockman 1989) and the ratios $N_{H e i} / N_{H i}=0.1$ and $N_{H e i i} / N_{H i}=0.01$. The best fit values for the simple absorbed power law model are summarized in Table 2 together with their $1 \sigma$ uncertainties for 1 interesting parameter. Two-parameter $\chi^{2}$ contour confidence levels at $\chi_{\text {min }}^{2}+1,+4.61$ and +5.99 - corresponding respectively to the $1 \sigma$ uncertainty for each single parameter and to the $90 \%$ and $95 \%$ joint confidence levels - are shown in Figure 2, 5 and 9 for the 1994, 1995 and 1996 data sets respectively.

\subsubsection{4 energy spectrum}

Our best fit flux in 1994 is slightly higher than the previous analysis of Fruscione et al., 1996 $\left(\mathrm{f}_{80 \AA}=6.44 \pm 0.33 \times 10^{-3}\right.$ photons $\mathrm{cm}^{-2} \mathrm{~s}^{-1}$ $\AA^{-1}$ compared to their $\mathrm{f}_{80 \AA}=290 \pm 15 \mu \mathrm{Jy}=$ $5.47 \pm 0.28 \times 10^{-3}$ photons $\left.\mathrm{cm}^{-2} \mathrm{~s}^{-1} \AA^{-1}\right)$ but our slope is within the errors $\left(\alpha=0.85_{-0.72}^{+0.66}\right.$ compared to their $\alpha=1.4 \pm 0.8)$. The $1 \sigma$ error bars reported 
in Fruscione et al., 1996 were not corrected for the effective area; the small difference in the value of the normalization can be possibly explained by the difference in the errors and a possible difference in the wavelength range used to model the spectrum. This is the only spectrum for which the addition of an absorbed interved gaussian line does not improve the fit (Tab.3).

\subsubsection{5 energy spectrum}

The 1995 EUVE DS/S observation was part of a large multiwavelength campaign during which a powerful TeV flare occured (Buckley et al. 1996). Simultaneously an X-ray flare developed (Takahashi et al. 1996), and the EUV followed with a short delay (Buckley et al. 1996). As illustrated in figure 3, the $E U V E$ observation spanned the flare $(\sim 104 \mathrm{ks})$, the decay $(\sim 218 \mathrm{ks})$ and the following quiescent state $(\sim 185 \mathrm{ks})$ The observation was performed $\sim 0.3^{\circ}$ off-axis and as expected the signal reaches down $\sim 70 \AA$. This spectral region is crucial since it covers the location of the wide EUV absorption feature proposed by K97 using less than half (the flare decay) of the 1995 data. In our analysis we corrected the data with the $\sim 0.3^{\circ}$ off-axis effective area (Marshall et al. 1999) and the resulting spectrum, binned over 1 $\AA$, is presented in Figure 4 .

In order to compare our data analysis to that of $\mathrm{K} 97$, and in an attempt to look for changes during the flare, we divided the 1995 observation into three parts (Figure 3):

Flare: April 25-28 ( 104ks), the time interval of the EUVE flare;

Decay: April 29 - May 6 ( $218 \mathrm{ks})$, the time interval analysed by K97;

Quiescence: May 7-13 ( $185 \mathrm{ks})$, the remaining data. The $E U V E$ spectra for the 3 parts are shown in Figiure 6 a,b and c.

Table 1 lists the exposures times and Table 2 summarizes the best-fit parameters for each one of the three parts. In Figure 7 we plot the twoparameter $\chi^{2}$ contour confidence levels.

\subsubsection{Comparison with previous results}

The 'decay' section of the 1995 spectrum deserves comparison with the analysis of K97, given the importance of the absorption line evident in their Figure 2. We show the effect of using the improper $\sim 0.3^{\circ}$ off-axis effective area in Figure 6b; we compared the EUVE spectrum obtained using the on-axis effective area (open cirles) as in $\mathrm{K} 97$, and the $0.3^{\circ}$ off-axis effective area (filled squares). The effect of using the appropriate calibration is to increase the flux below $\sim 80 \AA$ and so weaken the absorption feature $(\sim 45 \%)$. Another effect to consider is that K97 evaluated the level of the continuum in the EUV range by adopting an $\alpha_{\mathrm{EUVX}}(\approx 1.32)$ slope derived from the 0.15 kev EUV flux and the $1.5 \mathrm{keV} \mathrm{X}$-ray $(A S C A)$ flux, which resulted in a strong absorption feature (see also $\S 3$ for further discussion on this point).

Our best fit slope $\alpha=2.19 \pm 0.5$ (Table 2) is flatter than the $\alpha=3.5 \pm 0.8$ in K97. If we consider the same wavelength range (70-95 $\AA$ instead of 70$110 \AA$ ) ) we find $\alpha=2.54 \pm 0.56$, consistent with K97 result.

A simultaneous fit of an absorbed power law plus an absorbed (inverted) gaussian should provide a more reliable result. If the position of the gaussian is a free parameter then the reduced $\chi^{2}$ goes to 1.17 and a narrow $(\mathrm{FWHM}=1.8 \pm 0.5$ $\AA$ ) absorption feature is found at $\sim 72.7 \pm 0.3$ $\AA$ while the slope becomes significanlty flatter $(\alpha=1.62 \pm 0.59)$ (see Tab.3). This is the first time that an absorption feature in the EUV range is derived from EUV data alone.

Both for PKS 2155-304 (Konigl et al. 1995) and for Mrk 421 (K97) the presence of absorption features was previously derived on the basis of an extrapolation of the X-ray power law and not directly from $E U V E$ data. However there is strong evidence that the X-ray spectrum of the highenergy peaked BL Lac objects, including Mrk 421 , is concave (Sambruna et al. 1994, 1997; Tashiro et al. 1995; Takahashi et al. 1996a; Giommi et al. 1998; Guainazzi et al. 1999) and a gradual steepening with energy agrees with the "synchrotron self Compton" scenario, which has often been used to explain the spectral energy distribution of BL Lac objects (Ghisellini, Maraschi \& Treves 1985, Ghisellini 1989). Therefore, a simple power law model is an unreliable extrapolation from X-rays to the EUV.

In order to verify this assumption for Mrk 421 , we reanalyze, as an example, one of the many one-hour-long $1995 A S C A$ observations (the one on 25-26 April) undertaken simultaneously with the EUVE observation (1995 'flare'). This in or- 
der to investigate the spectral slope below 1.5 $\mathrm{keV}$ (Takahashi et al. 1996b and 1999 only published data for $1.5 \mathrm{keV}$ and above). First we fitted the $A S C A$ spectra with an absorbed broken power law and the best fit requires a break position at $E_{\text {break }}=1.77 \pm 0.07 \mathrm{keV}$ and the energy slopes varies from $1.44 \pm 0.16$ above the break to $0.98 \pm 0.02$ below the break (consistent with what found by Takahashi et al.). The broken power law model however does not give a good fit of the spectrum below $1.5 \mathrm{keV}$; a better result is found if we assume a "curved spectrum" parametrized as

$$
F(E)=E^{-\left(f(E) \alpha_{l o w}+(1-f(E)) \alpha_{h i g h}\right)}
$$

where $f(E)=\left(1-\exp \left(-E / E_{0}\right)\right)^{\beta}, \alpha_{\text {low }}$ and $\alpha_{\text {high }}$ are the low and high energy asymptotic slopes and $\beta$ is the curvature radius in the energy space. The best fit values for the curved model with fixed $\beta=1.0$ (e.g. Guainazzi et al. 1999) are $\alpha_{\text {low }}=0.57 \pm 0.06, \alpha_{\text {high }}=1.34 \pm 0.13$ and $E_{0}=$ $1.78 \pm 0.18$ (see fig. 10); these values show that the slope flattens toward lower energies and that an extrapolation with a simple power law is not an accurate way to predict the EUV continuum level; the low energy asymptotic slope $\left(\alpha_{\text {low }}=0.57\right)$ is generally in agreement with the flat slope derived from the EUV spectrum $(\alpha=0.71 \pm 0.61$ from Tab.3).

Note that in the case of PKS 2155-304 there is an additional problem: the ROSAT spectral slope used for the extrapolation was not measured simulteneously to the EUV spectrum and the spectral slope has been observed to harden when the source intensity increases (Giommi et al. 1998).

\subsubsection{6 energy spectrum}

In 1996 Mrk 421 was again observed $\sim 0.3^{\circ}$ offaxis with a total effective exposure of $354806 \mathrm{~s}$. The $1 \AA$ binned spectrum and the best fit parameters model are plotted in Figure 8; the corresponding contour confidence levels are shown in Figure 9.

\subsubsection{The EUV absorption feature}

We performed several fits using an absorbed power law model plus gaussian for all the observations. The results are listed in Table 3. In every case, except for the 1994 observation, the addition of a gaussian absorption line improves the fit. The power law slope is in the range $\alpha=[-0.15,1.62]$ which is not too distant from the slope measured from X-ray data, (see e.g. Guainazzi et al.with SAX data which gives $\alpha=0.3-1.1$ depending on the spectral model). The best-fit center of the gaussian absorption lines is in the 71-75 Årange with full width half maximum varying from 0.2 to $6 \AA$. We note however that the $6 \AA$ FWHM is relative to the global 1995 observation and it is probably due to the superposition of narrower slightly variable lines.

\section{Discussion}

\subsection{The absorption feature}

Ever since Einstein Pioneering observations suggested the existence of an ubiquitous absorption feature around $\sim 0.55 \mathrm{keV}$ in the $\mathrm{X}$-ray spectra of BL Lacertae objects (Canizares \& Kruper 1984, Madejski et al. 1991), much X-ray and EUV data has been collected with the goal of confirming the existence of such absorption features.

\subsubsection{EUV/X-ray absorption in other BL Lac objects}

The first object to show evidence of an X-ray absorption feature was PKS 2155-304 $(z=0.117)$ in a 1980 Einstein grating spectrum (Canizares \& Kruper 1984). The feature was detected at $\sim 0.6 \mathrm{keV}(\lambda=21 \AA)$ and extended for $50-100$ $\mathrm{eV}(\lambda=120-250 \AA)$; it was interpreted by the authors as O VIII Ly $\alpha$ emitted by high velocity material. Looking at a 1979 Einstein solid state spectrometer observation of the same object Madejski et al. 1991 found evidence for absorption at $\sim 0.55 \mathrm{keV}$, consistent with the grating result.

Krolik et al. 1985 modelled the absorption as due to an outflowing subrelativistic wind beamed in our direction.

PKS 2155-304 was subsequently observed by BBXRT in 1990 and the absorption feature at $\sim 0.55 \mathrm{keV}$ was confirmed in those data (Madejski et al. 1994), although instrumental effects cast some doubt on this result (Sambruna et al. 1997, Weaver et al. 1995).

In a long exposure ( $>100 \mathrm{ks})$ Beppo-SAX spectrum of PKS 2155-304 in the soft X-rays (Giommi et al. 1998) the best fit model is improved (at $99 \%$ confidence) when a notch at $0.55 \pm 0.04 \mathrm{keV}$ 
is added to the model, although the magnitude of the feature is close to the instrument calibration limits.

PKS 2155-304 was also observed by EUVE. A 199230 ks observation gives hints of an absorption feature at $\sim 80 \AA$ (Fruscione et al. 1994). Two longer observations in June $(130 \mathrm{ks})$ and in July (150 ks) 1993 suggested the existence of 2 to 5 absorption features at a $\geq 3 \sigma$ level in the range 75 to $110 \AA$ (Konigl et al. 1995). Extrapolating the ROSAT X-ray spectral slope into the EUV the authors found evidence of a wide absorption feature from $\sim 75$ to $\sim 85 \AA$ and interpreted it as the superposition of Doppler-smeared absorption lines (L-shell transitions of $\mathrm{Mg}$ and $\mathrm{Ne}$ as well as M-shell transitions of $\mathrm{Fe}$ ) that originate in a high-velocity clumped outflow from the nucleus. According to this model the X-ray line spectrum should be dominated by an O VII $\mathrm{K} \alpha$ broadened and blueshifted feature. This corresponds to the feature observed in by Einstein and in 1990 by $B B X R T$, but the predictions are an order of magnitude greater than the observations; to match BBXRT measurement the oxygen must be present in subcosmic abundance (Konigl et al. 1995).

Another BL Lac object to show an X-ray absorption feature is H1426+428 $(z=0.129)$. Sambruna et al. (1997) reanalyzed a BBXRT 1990 observation and found a broad feature around $0.5-0.6 \mathrm{keV}$ confirmed by an ASCA 1994 spectrum, but not by a ROSAT 1993 spectrum.

A third BL Lac, PKS 0548-322 $(z=0.069)$ (Sambruna \& Mushotzky, 1998) confirmed the early discovery of an absorption feature from the Einstein spectrum (Madejski et al. 1991); this feature is present in $A S C A$ data and can be modeled with either an edge at $\sim 0.66 \mathrm{keV}$ and optical depth $\tau \sim 0.3$ or with a notch at $\sim 0.82 \mathrm{keV}$ and fixed width of $\Delta E=0.1 \mathrm{keV}$ and covering fraction $f_{c} \sim 0.1$. The $A S C A$ absorpton feature is at energies significantly higher than in previous X-ray observations, when the continuum was in a slightly higher state (by a factor $\sim 1.4$ ); this provides tentative evidence for variability in the absorption feature.

\subsection{2. $E U V / X$-ray absorption in Markarian 421}

K97 interpret the absorption feature in the context of the Konigl et al. (1995) model for PKS 2155-304 as a superposition of Mg VIII and
Mg IX absorption lines. The implied ionization state of the gas would require stronger O VII absorption in the X-ray range than is allowed by observations and the authors invoke subcosmic oxygen abundance to explain its absence.

Our analysis of the 1994, 1995 and 1996 EUVE spectra of Mrk 421 shows an absorption feature inferred directly from the $E U V E$ data, (i.e. by modelling the EUV continuum and the absorption line simultaneously). The simplest model for this feature is a reverted Gaussian at about 71-75 $\AA$ a maximum FWHM of the order of few $\AA$.

In order to investigate likely absorption features in a BL Lac environment, we ran photoionization equilibrium models (Nicastro et al. 1999, Nicastro et al. 2000) which include the strongest 300 emission and resonant absorption lines down to $50 \mathrm{eV}$ (oscillator strength $>0.1$ ). We find that the predicted wavelengths and strengths of the absorption lines, both absolute and relative, are highly sensitive to the properties of the photoionizing continuum and the absorbing gas (e.g. the ionization state and the assumed outflowing velocity). The number of transitions in the model also has a strong effect. However assuming the observed EUV-to-X-ray continuum and adjusting the gas paramters to maximize the relative abundance of OVII $(\sim 65 \%)$ both an OVII K $\alpha$ absorption line $(\mathrm{EW}=0.25 \AA)$ and a FeIX L $\left(\lambda_{\text {rest }}=82.43\right.$ $\AA, \mathrm{EW}=0.2 \AA$ ) absorption line are expected respectively around $0.6 \mathrm{keV}$ and $73 \AA$ (assuming an outflow velocity of $0.15 \mathrm{c}$ ). The equivalent width of the lines is too small to give significant detections, however, especially in the EUV regime, the presence of many fainter satellite lines (not included in the model) could explain the observed feature For example between the Si VI line $(\lambda=72.34 \AA)$ and the Mg VI complex $(\lambda 71.3 \AA)$, both included in the model, at least 15 additional lines from ionized $\mathrm{Fe}, \mathrm{Al}, \mathrm{Be}$ and $\mathrm{Ne}$ are not included (oscillator strength 0.01-0.1) and could be important especially if the elements are abundant.

Even though no strong absorption line seem to be visible in the X-ray spectrum, we note that both in the 1979 Einstein data (Madejski et al. 1991) and in the 1997 and 1998 Beppo-SAX spectrum (Guainazzi et al. 1999, Fossati et al. 2000) the fit improves when adding an absorption feature centered at $\sim 0.55 \mathrm{keV}$.

In order to investigate any possible correlation 
between spectral changes and source flux, we plotted in Fig. 11 the energy index as a function of total EUV flux for the the power law plus gaussian models. However the large error bars prevent any statististically significant conclusion.

\section{Conclusions}

From EUVE data, we have found a clear absorption feature at $\sim 71-75 \AA$ in the spectrum of MRK 421. The absorption features detected in the EUV/X-ray spectra of BL Lac objects are often poorly determined statistically, as in the BeppoSAX observations of PKS 2155-304 and MRK 421 (Giommi et al. 1998 and Guainazzi et al. 1999), but it is interesting to notice that different instruments on different satellites point to the same possible $0.55 \mathrm{keV}$ feature and to a (probably linked) absorption feature in the EUV spectra of the same objects.

We do not know if X-rays and EUV absorption features are present in all or many BL Lac objects, as initially suggested by Madejski et al. 1991, but to-date absorption features at $0.5-0.6 \mathrm{keV}$ have been found - or at least statistically improves the spectral fitting - in four of the brightest BL Lacs objects (PKS 2155-304, H1426+428, PKS 0548322 and MRK 421) and EUV absorption features has been found in two of them (PKS 2155-304 and MRK 421). Future observations covering the EUV to X-ray energy bands with much higher resolution and collecting area, as with Chandra and XMM, will soon be able to answer this question and give more detailed observational data on which to develop new models.

We would like to thank Dr. Herman Marshall for providing the off-axis effective areas for the EUVE DS instrument, and Drs. I. Papadakis, A. Celotti, R. Sambruna, M. Elvis for the useful discussions. We are particularly grateful to F. Nicastro and G. Matt for making their models available to us. We thank the EUVE science team and in particular Dr. Roger Malina for their logistical support for our continuing analysis effort on $E U V E$ data. This research has made extensive use of the High Energy Astrophysics Science Archive Research Center Online Service (HEASARC), provided by the NASA-Goddard Space Flight Center, of the NASA/IPAC Extragalactic Database
(NED) operated by the Jet Propulsion Laboratory, Caltech, under contract with NASA and of NASA's Astrophysics Data System Abstract Service. A.F. and I.C. are grateful to the Brera Observatory for the ospitality. This work was supported by AXAF Science Center NASA contract NAS 8-39073 and by NASA grants NAG 5-3174 and NAG 5-3191.

\section{REFERENCES}

Abbott, M. et al. 1996, ApJS, 107, 451

Bowyer, S.\& Malina, R. F., 1991, in Extreme Ultraviolet Astronomy, ed. R.F. Malina \& S. Bowyer (New York: Pergamon Press), 397

Buckley, J. H. et al. 1996, ApJ, 472, 9

Cagnoni, I., Fruscione, A. \& Papadakis, I. E., (paper II) 1999, this volume

Canizares, C. R. \& Kruper, J., 1984, ApJ, 278, 99

lvis, M., Wilkes, B. J. \& Lockman, F. J., 1989, AJ, 97, 777

Fossati, G. et al., 2000, ApJ in press

Fruscione, A. 1996, ApJ, 459, 509

Fruscione, A., Bowyer, S., Konigl, A. \& Kahn, S. M., 1994, ApJ, 422, 55

Fruscione, A., Bruhweiler, F., Cheng, K.P., Hall, C.R., kafatos, M., Ramos, E. \& Kondo, Y., 1996, in UV and X-ray Spectroscopy of Astrophysical and Laboratory Plasmas, ed. K. Yamashita \& T. Watanabe (Tokyo: Universal Academic Press), 351

George, I.M., Warwick, R. S. \& Bromage, G. E., 1988 MNRAS 232, 793

George, I. M., Warwick, R. S. \& McHardy, I. M., 1988, MNRAS, 235, 787

Ghisellini, G., 1989, MNRAS, 236, 341

Ghisellini, G. Maraschi, L. \& Treves, A., 1985, A\&A 146, 204

Giommi, P., Barr, P., Garilli, B., Maccagni, D. \& Pollock, T., 1990, ApJ, 356, 432

Giommi, P. et al. 1998, A\&A, 333, L5 
Guainazzi, M., Vacanti, G., Malizia, A., O'Flaherty, K. S., Palazzi, E. \& Parmar, A. N., 1999, A\&A, 342, 124

Hettrick, M.C. \& Bowyer, S., 1983, Appl. Opt., 3921

Kartje, J.F., Konigl, A., Hwang, C.Y. \& Bowyer, S., 1997, ApJ, 474, 630 (K97)

Kerrick, A. D. et al. 1995, ApJ, 438, 59

Konigl, A., Kartje, J. F., Bowyer, S., Kahn, S. M. \& Hwang, C.-Y., 1995, ApJ, 446, 598

Krolik, J. H., Kallman, T. R., Fabian, A. C. \& Rees, M. J., 1985, ApJ, 295, 104

Macomb, D. J. et al. 1995, ApJ, 449, 99

Madejski, G. M., Mushotzky, R. F., Weaver, K. A., Arnaud, K. A. \& Urry, C. M., 1991, ApJ, 370,198

Madejski, G. M. et al. 1994, in "BBXRT, a preview to astronomical X-ray spectroscopy in the 90's", ed. P. Serlemitsos \& S. J. Shrader, Greenbelt: NASA, 63

Marshall, H. L., Fruscione, A. \& Carone, T. E., 1995, ApJ, 439, 90

Marshall, H. L. et al. 1999 in preparation

Makino, F. et al. 1987, ApJ, 313, 662

Malina, R. et al. 1994, AJ, 107, 751

Maza, J., Martin, P. G. \& Angel, J.R.P., 1978, ApJ, 224, 368

Morrison, R. \& McCammon, D., 1983, ApJ, 270, $119-122$

Mufson, S.L., Hutter, D.J. \& Kondo, Y., 1989, in "BL Lac Objects": proceedings of a workshop held in Como, Italy, September 20-23, 1988. Editors, L. Maraschi, T. Maccacaro, M.-H. Ulrich; Publisher, Springer-Verlag, Berlin, New York, NY, 1989, p.341

Mufson, S.L., Hutter, D.J., Kondo, Y., Urry, C. M. \& Wisniewski, W. Z., 1990 ApJ, 354, 116

Mushotzky, R. F., Boldt, E. A., Holt, S. S. \& Serlemitsos, P. J., 1979, ApJ, 232, 17
Nicastro F., Fiore F. and Matt G., 1999, ApJ, 517, 108

Nicastro F., Elvis M., Fiore F., Matt G. and Savaglio S., 2000, Proceedings of "X-ray Astronomy '99", 1999, September 6-10, Bologna, Italy, astro-ph/0007050

Pounds, K. A. et al. 1993, MNRAS, 260, 77

Punch, M. et al. 1992, Nature, 358, 477

Pye, J. P. et al. 1995, MNRAS, 274, 1165

Richetts et al. 1976, Nature, 259,546

Rumph, T., Bowyer, S. \& Vennes, S., 1994, AJ, 107, no. 6, 2108-2114

Sambruna, R. M., Barr, P., Giommi, P., Maraschi, L., Tagliaferri, G. \& Treves, A., 1994, ApJS, 95, 371

Sambruna, R. M. et al. 1997, ApJ, 483, 774

Sambruna, R. M. \& Mushotzky, R. F., 1998, ApJ, 502,630

Sirk, M.M., internal EUVE memo MMS/EUVE/0084/94

Sirk, M.M., Vallerga, J.V., Finley, D.S., Jelinsky, P. \& Malina, R.F., 1997, ApJS, 110, 347

Takahashi, T et al. 1996a, 1996, ApJ, 470, 89

Takahashi, T. et al. 1996b, Mem. SaIt. 67, 533T

Takahashi, T., Madejski, G. \& Kubo, H., 1999, ApJ, 11, 177

Tashiro, M., Makishima, K., Ohashi, T., IndaKoide, M., Yamashita, A., Mihara, T. \& Kohmura, Y., 1995, PASJ, 47, 131

Ulrich, M.-H., Kinman, T. D., Lynds, C. R., Rieke, G. H., \& Ekers, R. D 1975, ApJ, 198, 261

Welsh, B.Y., Vallerga, J.V., Jelinsky, P., Vedder, P.W., Bowyer, S. \& Malina, R.F., 1990, Opt. Eng., 29, 752

Zhang, F. J. \& Baath, L. B., 1990, A\&A, 236, 47

This 2-column preprint was prepared with the AAS LATEX macros v5.0. 
Fig. 1.- EUV spectrum of MRK 421 obtained in 1994 with the SW spectrometer. The best fit power law model is overplotted (solid line). The lower panel shows the residuals to the fit.

Fig. 2.- $\chi^{2}$ contour confidence levels for normalization and energy index of the 1994 spectrum. The contours correspond to $\chi_{\min }^{2}+1,+4.61$ and +5.99 , respectively the $1 \sigma$ uncertainty for each single parameter and the $90 \%$ and $95 \%$ joint confidence levels. The best fit is marked by the filled symbol.

Fig. 3.- April 25 - May 131995 Deep Survey lightcurve binned over one average EUVE orbit ( 5544 s). The "flare", "decay" and "quiescence" intervals are marked.

Fig. 4.- EUV spectrum of MRK 421 obtained in 1995 with the SW spectrometer during a $0.3^{\circ}$ offaxis observation. Hence note the increased coverage toward shorter wavelengths. The best fit power law model (dotted line) and the best fit power law + inverted gaussian model (solid line) are shown together with the residuals to the fits.

Fig. 5.- Same as Fig. 2 for the 1995 spectrum and the power law model.

Fig. 6.- Individual sections of the 1995 spectrum of MRK 421 (a) During the flare (MJD 4983249835 or Apr. 25-28); (b) during April 29 - May 6(MJD 49835-49843) for direct comparison with the analysis by Kartje et al. 1997; (c) during the remaining period, May 7-13 (MJD 49844-49851). The best fit power law model (dotted line) and the best fit power law + inverted gaussian model (solid line) are shown. The open circles in (b) correspond to the spectrum corrected with the onaxis effective area (not accurate for this off-axis observation) as presented in Kartje et al. 1997.

Fig. 7.- Same as in Fig. 2 for the three sections of the 1995 observation and the power law model. They are displayed, from the top to the bottom, in temporal order: part 1 corresponds to the flare, part 2 to the decay and part 3 to the quiescent period.

Fig. 8.- EUV spectrum of MRK 421 obtained in 1996 with the SW spectrometer during a $0.3^{\circ}$ off-axis observation. The best fit power law model (dotted line) and the best fit power law + inverted gaussian model (solid line) are shown.

Fig. 9.- Same as in Fig. 2 for the 1996 spectrum and the power law model

Fig. 10.- ASCA spectrum of MRK 421 obtained in 1995 during the flare. The solid lines represent the best-fit model when an absorbed power-law with a gradually changing spectral index is applied to the spectrum of all the instruments simultaneously (upper panel ). The lower panel shows the residuals.

Fig. 11.- EUV source flux vs. energy index $(\alpha)$ for the power law component of the power law plus gaussian model. 
TABLE 1

EUVE OBSERVATIONS OF MrK 421

\begin{tabular}{|c|c|c|c|c|c|c|}
\hline Year & Instrument $^{\mathrm{a}}$ & Start Date & End Date & $\begin{array}{l}\mathrm{T}_{\exp } \mathrm{b} \\
(\mathrm{ks})\end{array}$ & $\begin{array}{c}\text { Count rate }^{\mathrm{c}} \\
\left(\mathrm{c} \mathrm{s}^{-1}\right)\end{array}$ & Spectral Data \\
\hline $1994^{\mathrm{d}}$ & $\mathrm{DS} / \mathrm{S}$ & April 2 & April 12 & 280 & 0.18 & yes \\
\hline 1995 & Scanner & Feb. 4 & Feb. 7 & 68 & 0.45 & no \\
\hline 1995 & $\mathrm{DS} / \mathrm{S}$ & April 25 & May 13 & 355 & 0.29 & yes \\
\hline 1995 (flare) & $\mathrm{DS} / \mathrm{S}$ & April 25 & April 28 & 86 & 0.37 & yes \\
\hline 1995 (decay) $^{\mathrm{e}}$ & $\mathrm{DS} / \mathrm{S}$ & April 29 & May 6 & 154 & 0.29 & yes \\
\hline 1995 (quiescence) & $\mathrm{DS} / \mathrm{S}$ & May 7 & May 13 & 115 & 0.22 & yes \\
\hline 1996 & $\mathrm{DS} / \mathrm{S}$ & April 17 & April 30 & 299 & 0.30 & yes \\
\hline 1996 & $\mathrm{DS} / \mathrm{S}$ & May 10 & May 11 & 3.6 & 0.30 & no \\
\hline 1997 & Scanner & Feb. 7 & Feb. 11 & 108 & 0.27 & no \\
\hline $1998^{f}$ & $\mathrm{DS} / \mathrm{S}$ & April 19 & May 1 & $\ldots$ & $\ldots$ & $\ldots$ \\
\hline
\end{tabular}

a "DS/S" indicates that the source was observed simultaneously in the $3 E U V E$ spectrometers and in the Deep Survey photometer. "Scanner" indicates that the source was observed simultaneously in the three EUVE photometers during a pointing within the Right Angle Program.

${ }^{\mathrm{b}}$ Total exposure time calculated eliminating (i) all SAA passages (ii) all satellite daytime data (iii) all times during which the detector was turned off (iv) all times affected by possible earth blockage. It does include corrections for telescope vignetting, deadtime and limited telemetry allocation (primbshing).

${ }^{\mathrm{c}}$ Average count rate in the Lexan/B filter $(\approx 60-180 \AA)$. The count rate in the scanner has been normalized to the one in the DS assuming an average spectral shape, to take into account the difference in the effective area between the two instruments.

${ }^{d}$ This observation was presented in Fruscione et al. (1996

e This part of the observation was presented in Kartje et al. 1997)

${ }^{\mathrm{f}}$ This observation is not included in this paper 
TABLE 2

Spectral fits to the EUV SPeCtrum of MRK 421 assuming an absorbed Power LaW model.

Fixed parameters ARe $N_{H}=1.45 \times 10^{20} \mathrm{CM}^{-2}, N_{H e I} / N_{H I}=0.1$ AND $N_{H e I I} / N_{H I}=0.01$

\begin{tabular}{|c|c|c|c|c|c|}
\hline Obs. & & $\begin{array}{c}\text { Range } \\
(\AA)\end{array}$ & $\alpha$ & $\begin{array}{c}10^{-3} f_{80 \stackrel{\circ}{\mathrm{a}}}\left(\text { phot } \mathrm{cm}^{-2} \mathrm{~s}^{-1} \AA^{-1}\right)\end{array}$ & $\chi_{\nu}^{2}$ \\
\hline 1994 & & $75-110$ & $0.85_{-0.72}^{+0.66}$ & $6.44 \pm 0.33$ & 1.06 \\
\hline 1995 & & $70-110$ & $1.13 \pm 0.38$ & $6.70 \pm 0.22$ & 2.45 \\
\hline Flare & Apr. 25-28 & $70-110$ & $1.43 \pm 0.56$ & $10.28 \pm 0.51$ & 1.57 \\
\hline Decay & Apr. 29-May 6 & $70-110$ & $2.19 \pm 0.50$ & $7.24 \pm 0.33$ & 1.24 \\
\hline Quiescence & May 7-13 & $70-110$ & $-0.60_{-1.16}^{+1.13}$ & $3.53 \pm 0.35$ & 1.47 \\
\hline 1996 & & $70-110$ & $2.12 \pm 0.31$ & $9.32 \pm 0.27$ & 1.61 \\
\hline
\end{tabular}


TABLE 3

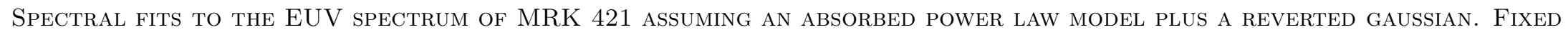
PARAMETERS AS IN TABLE 2

\begin{tabular}{|c|c|c|c|c|c|c|c|c|}
\hline \multirow[b]{2}{*}{ Obs. } & & \multicolumn{2}{|c|}{ Power Law } & \multicolumn{3}{|c|}{ Gaussian } & \multirow[b]{2}{*}{$\chi_{\nu}^{2}$} & \multirow[b]{2}{*}{$\begin{array}{c}\text { Flux }(70-110 \AA) \\
\left(10^{-3} \frac{\mathrm{phot}}{\mathrm{cm}^{2} \mathrm{~s}}\right)\end{array}$} \\
\hline & & $\alpha$ & $\left(10^{-3} \frac{\mathrm{f}_{\mathrm{phot}}}{\mathrm{cm}^{2} \mathrm{sA}}\right)$ & $\begin{array}{l}\lambda_{0} \\
(\AA)\end{array}$ & $\begin{array}{c}\text { FWHM } \\
(\AA)\end{array}$ & $\begin{array}{c}\text { Ampl. } \\
\left(10^{-3} \frac{\mathrm{phot}}{\mathrm{cm}^{2} \mathrm{sA}}\right)\end{array}$ & & \\
\hline 1994 & & $-0.29 \pm 0.83$ & $6.72 \pm 0.35$ & $79.3_{-0.07}^{+1.05}$ & $0.20 \pm 0.05$ & $-10.67 \pm 0.95$ & 1.14 & $5.06^{\mathrm{a}}$ \\
\hline 1995 & & $1.14 \pm 0.41$ & $7.55 \pm 0.22$ & $73.5 \pm 0.84$ & $6.07 \pm 1.47$ & $-2.23 \pm 0.48$ & 1.17 & 8.86 \\
\hline Flare & Apr. 25-28 & $0.71 \pm 0.61$ & $11.6 \pm 0.52$ & $74.87 \pm 0.45$ & $3.04 \pm 0.95$ & $-5.86 \pm 0.14$ & 0.57 & 14.11 \\
\hline Decay & Apr. 29-May 6 & $1.62 \pm 0.59$ & $7.84 \pm 0.34$ & $72.75 \pm 0.28$ & $1.86 \pm 0.51$ & $-5.22 \pm 1.30$ & 1.17 & 9.33 \\
\hline Quiescence & May 7-13 & $0.63 \pm 1.29$ & $3.90 \pm 0.35$ & $71.43 \pm 0.04$ & $0.17_{-0.06}^{+0.23}$ & $-6.55 \pm 3.17$ & 1.29 & 4.83 \\
\hline 1996 & & $-0.15 \pm 0.38$ & $9.64 \pm 0.28$ & $71.82_{-0.60}^{+0.06}$ & $0.66 \pm 0.12$ & $-10.40 \pm 2.75$ & 1.10 & 12.02 \\
\hline
\end{tabular}

aThis is calculated in the $75-110 \AA$ range for the model in Table 2 